\title{
Propuesta integrativa teórico-práctica utilizando la metodología "just in case" en la asignatura de Entrenamiento Deportivo I (GCCAFD)
}

\section{A theoretical-practical integrative learning proposal using the "just-in-case" method in the Sports Training subject (Sports Science Degree)}

ALEJANDRO MUÑOZ LóPEZ

ORCID: https://orcid.org/0000-0003-1206-8697

Departamento de Motricidad Humana

y Rendimiento Deportivo

Universidad de Sevilla

amunoz26@us.es

Fecha de recepción: 19/11/2019

Fecha de aceptación: 22/11/2019

DOI: http://dx.doi.org/10.12795/9788447221912.098

Pp.: 2204-2222 
El objetivo de este Ciclo de Mejora Docente fue el desarrollo teórico-práctico de un bloque de contenidos en la asignatura de Entrenamiento Deportivo I, del Grado en Ciencias de la Actividad Física y del Deporte en la Universidad de Sevilla. Durante 2 semanas, los alumnos participantes realizaron una secuencia de actividades siguiendo la metodología de trabajo just in case. Los alumnos debían estudiar los apuntes en casa para posteriormente en clase, resolver problemas planteados por el profesor. La evaluación inicial mostró un bajo nivel de comprensión en relación a los contenidos principales del tema. Tras la aplicación del ciclo, se observó una gran evolución del grupo. Incluso en la pregunta más dificil, mas del 50\% del grupo pasó del corte de calificaciones inferiores (A y B) hacia calificaciones superiores (C y D). En conclusión, la aplicación de un Ciclo de Mejora Docente en la asignatura de Entrenamiento Deportivo I ha ayudado a comprender mejor cuestiones habitualmente aprendidas de forma errónea, así como a enseñar de una forma diferente a las clases magistrales habituales.

Palabras clave: Entrenamiento Deportivo I, Grado Ciencias de la Actividad Física y del Deporte, Docencia universitaria, Experimentación Docente Universitaria, Clase Magistral.

\section{Abstract}

The purpose of this Teaching Improvement Cycle was to develop a theorical-practical content block in the Sports Training subject of the Sports Science Degree at the University of Seville. Along 2 weeks, the students participated in a sequence of activities following the just in case learning method. Participants had to study themselves the theoretical concepts for later during lessons with the professor use the time for resolving quizzes. The initial test showed a low comprehension level related to the main contents. After the Cycle application, it was observed a great group improvement. Indeed, at the most difficult question, more than $50 \%$ of the group move from lower qualifications ( $A$ and $B$ ) to higher qualification ( $C$ and $D)$. In conclusion, the application of a Teaching Improvement Cycle in the Sports Training subject helped to a better comprehension of the issues usually learned wrongly and to teach in a different way from the usual master classes.

Keywords: Sports Training, Sports Science Degree, University Teaching, University Teaching Research, Master Class. 


\section{Contexto}

El siguiente Ciclo de Mejora Docente (CMD) trata sobre el desarrollo de los contenidos teóricos y prácticos relacionados con los medios y metodologías del entrenamiento de fuerza, en la asignatura de Entrenamiento Deportivo I en el Grado de Ciencias de la Actividad Física y del Deporte. Dicho grado pretende la preparación de los estudiantes hacia una serie de salidas profesionales muy concretas, entre las que destaca el entrenamiento. Aunque existen materias que están relacionadas y esta asignatura comienza en tercero de carrera, es la primera que comienza a tratar con cierta especialización diversos temas específicos en relación con esta salida profesional.

La mayoría de los estudiantes poseen ciertos conocimientos en entrenamiento, bien sea porque a lo largo de los primeros cursos de la carrera siempre suelen hacerse referencia a conceptos relacionados en otras asignaturas, o bien porque tienen o han tenido experiencias en relación con él (es habitual que este perfil de alumnado practique deporte, por lo que eminentemente habrán entrenado en algún momento de su vida de una forma u otra). Sin embargo, debido a la falta de especialización y conocimientos específicos que existen en muchos profesionales de nuestro sector (intrusismo, etc...), estos conceptos suelen estar en gran confusión, por lo que habitualmente los estudiantes comienzan esta asignatura con una serie de conocimientos previos muy genéricos, pero, sobre todo, mezclados y confundidos entre sí.

Así, nos encontramos ante un contexto de confusión de experiencias previas que deben ser reconducidas, a la vez que nuevos conocimientos son incluidos, buscando la especificidad de los contenidos relacionados con la materia. Más en concreto, esta asignatura versa principalmente sobre el conocimiento y la clasificación de las principales cualidades motrices, entre los que destacan la fuerza, la 
resistencia, la coordinación y la amplitud de movimiento. De todos estos, es la capacidad de ejercer fuerza la más importante de todas (sin fuerza, no existe movimiento). Además, es uno de los conjuntos de contenidos que más confusión acarrea, debido a la alta especialidad que requiere poder llegar a entender, incluido a nivel básico, el funcionamiento y la respuesta del organismo humano a estímulos de entrenamiento relacionado.

Por ello, este CMD está orientado específicamente a los contenidos relacionados con el entrenamiento de fuerza. En contenidos anteriores, los estudiantes han recibido información sobre esta capacidad motriz y sus diferentes clasificaciones. En esta ocasión, los contenidos se centran sobre los medios y métodos para poder desarrollar esta capacidad. Por experiencia, los estudiantes confunden entre medios y métodos y, además, les es dificil conocer las agrupaciones de los mismos y las bases que los distinguen. Esto es muy importante porque, en un futuro, conociendo estas clasificaciones y el por qué de las mismas, podrán llegar a entender cualquier otro nuevo medio o método que se desarrolle, conociendo así sus efectos y entendiendo el por qué deben seleccionar y aplicar cada cosa en cada momento, lo que claramente les debe diferenciar de cualquier otro tipo de profesional que no sea de las Ciencias de la Actividad Física y del Deporte.

Por último, este CMD incluye la metodología just in time. Este grupo de estudiantes ya han experimentado durante dos semanas anteriores este tipo de metodología. Sin embargo, no están para nada acostumbrados a metodologías activas (así nos lo hacen saber) y, sobre todo, aunque la mayoría de los alumnos optan desde primero por un sistema de evaluación por curso, para nada están acostumbrados a un trabajo diario o semanal sobre cada asignatura, lo que en nuestra opinión es un gran error. En concreto, la metodología just in time, ideada por Gregor Novak (Universidad de Purdue, Indiana), pretende 
fomentar el estudio previo de los alumnos antes de clase, así como la comunicación de sus dificultades y dudas al profesor (Prieto Martin et al., 2018). En concreto, esta forma de orientar la enseñanza recibe este nombre porque el profesor replantea su clase a partir de las necesidades de comprensión que detecta en sus alumnos (justo a tiempo). Así, además de poder resolver las dificultades e incluir nudos de aprendizaje en el día a día, se obtiene un mayor tiempo para realizar reflexiones en clase, además de incluir a los estudiantes en su propio proceso de enseñanza aprendizaje.

\section{Diseño previo del CMD}

Este CMD ha sido llevado a cabo durante un total de 2 semanas del curso 2019-20, durante 10 horas lectivas. En él, se incluyen desde actividades de preparación de los contenidos y aplicación del mismo, hasta evaluación del proceso.

\section{Mapa de contenidos}

Los contenidos a tratar durante este CMD están representados en la Figura 2. Como se observa, se dividen en dos partes fundamentales, en torno a una pregunta central: "cómo hacer un entrenamiento de fuerza". Esta pregunta es el eje fundamental de los contenidos a tratar, la cual requiere de dos conocimientos fundamentales para poder resolverla; 1 ) qué debo modificar (metodología) y 2) con qué lo hago (medios). 


\section{TEMA 6}

MÉTODOS

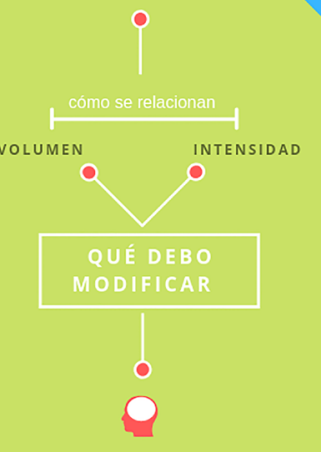

OBJETIVOS DEL

ENTRENAMIENTO
CÓMO HAGER UN ENTRENAMENTO DEFOEPAA

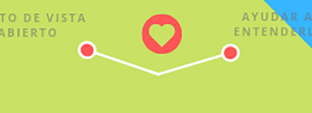

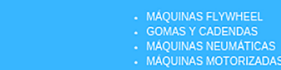

MEDIOS MODERNOS

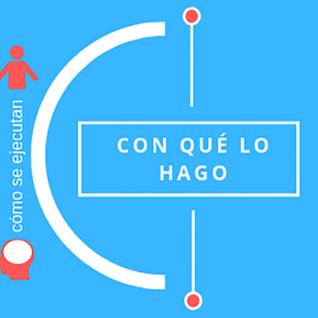

MEDIOS TRADICIONALES

ISOMEIRICOS
PESOS LIBRES

. PESOS LIBRES
MAQUINAS DEPL

Figura 1. Mapa de contenidos del CMD. En rojo, la clasificación de los tipos de contenidos (conceptuales, procedimentales y actitudinales)

\section{Modelo metodológico}

La Figura 1 representa el modelo metodológico seguido. Se pueden observar claramente diversas fases. De mayor a menor importancia, encontramos las formas de mayor a menor tamaño. En primer lugar, se realizará una evaluación (ver Cuestionario Evaluación en apartado 2.4) con el objetivo de conocer en qué forma los estudiantes tienen asimilados los contenidos principales relacionados con el CMD. Dicha evaluación será también realizada al final del ciclo. Siguiendo las líneas del estilo de enseñanza de este ciclo, los estudiantes estudiarán el temario preparado previamente por el profesor, entregado a través de la plataforma aula virtual en formato digital. Los estudiantes dispusieron de un plazo de una semana para estudiar la mitad del temario y rellenar el denominado cuaderno de apuntes, lo que posteriormente sirve de guía para la fase 
de Dudas (Figura 1, D). Tras este tramo, se plantea un problema relacionado con la temática para resolver, con posterior reflexión (Figura 2, RP y R). De forma seguida a la intervención en el aula, se realiza una vivencia práctica con su consiguiente reflexión final (Figura 1, VP y R). Cada intervención en aula y en campo se realizará en dos bloques diferenciados en dos semanas diferentes, correspondientes cada semana a la mitad de todos los contenidos (Figura 1).

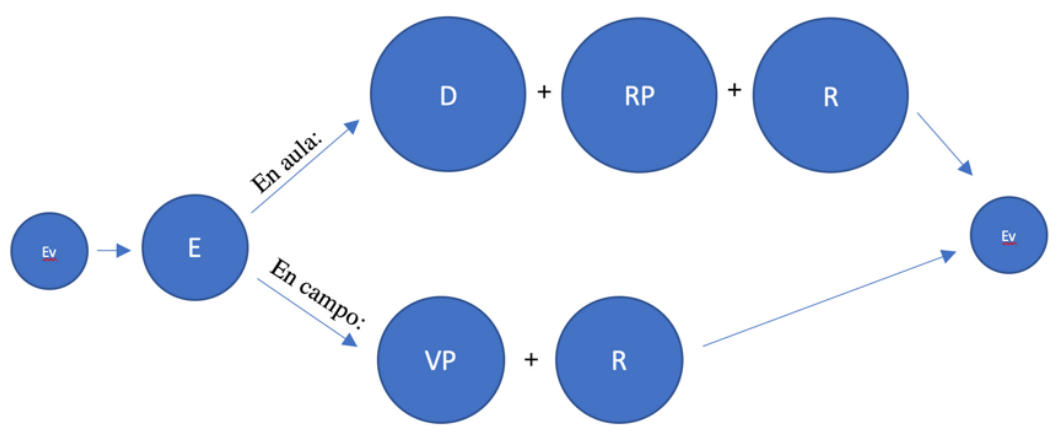

Figura 2. Modelo metodológico. Ev= evaluación. $E=$ estudio previo del temario. $\mathrm{D}=$ resolución dudas. $\mathrm{RP}=$ resolución de problemas. $\mathrm{R}=$ reflexión. $\mathrm{VP}=$ vivencia práctica.

\section{Actividades a desarrollar}

Más en concreto, esta propuesta de contenidos será llevada a cabo a través de unas actividades específicas, en relación con las fases del modelo metodológico (Figura 1). En la Tabla 1 se resumen cada una de las actividades, así como la temporalización de las mismas.

Tabla 1. Secuencia de actividades

\begin{tabular}{|l|l|l|l|}
\hline Temporalización & Fase del modelo & Actividad & $\begin{array}{l}\text { Duración } \\
\text { presencial }\end{array}$ \\
\hline Semana 1 & Evaluación & Evaluación inicial & 30 minutos \\
\hline
\end{tabular}

Jornadas de Formación e Innovación Docente del Profesorado | № 2 (2019) Esta obra se distribuye con la licencia Creative Commons Reconocimiento-NoComercial-SinObraDerivada 


\begin{tabular}{|c|c|c|c|}
\hline & \multirow{2}{*}{$\begin{array}{l}\text { Estudio previo del } \\
\text { temario }\end{array}$} & Estudio del temario I & No presencial \\
\hline & & $\begin{array}{l}\text { Cuaderno de } \\
\text { apuntes I }\end{array}$ & No presencial \\
\hline \multirow{3}{*}{ Semana 2} & \multirow{2}{*}{$\begin{array}{l}\text { Intervención en } \\
\text { aula }\end{array}$} & $\begin{array}{l}\text { Resolución de dudas } \\
\text { I }\end{array}$ & 45 minutos \\
\hline & & $\begin{array}{l}\text { Resolución y } \\
\text { reflexión problema I }\end{array}$ & 45 minutos \\
\hline & $\begin{array}{l}\text { Intervención en } \\
\text { campo }\end{array}$ & $\begin{array}{l}\text { Vivencia práctica y } \\
\text { reflexión I }\end{array}$ & 2 horas \\
\hline \multirow[b]{2}{*}{ Semana 2-3 } & \multirow{2}{*}{$\begin{array}{l}\text { Estudio previo del } \\
\text { temario }\end{array}$} & Estudio del temario II & No presencial \\
\hline & & $\begin{array}{l}\text { Cuaderno de apuntes } \\
\text { II }\end{array}$ & No presencial \\
\hline \multirow{3}{*}{ Semana 3} & \multirow{2}{*}{$\begin{array}{l}\text { Intervención en } \\
\text { aula }\end{array}$} & $\begin{array}{l}\text { Resolución de dudas } \\
\text { II }\end{array}$ & 45 minutos \\
\hline & & $\begin{array}{l}\text { Resolución y } \\
\text { reflexión problema II }\end{array}$ & 45 minutos \\
\hline & $\begin{array}{l}\text { Intervención en } \\
\text { campo }\end{array}$ & $\begin{array}{l}\text { Vivencia práctica y } \\
\text { reflexión II }\end{array}$ & 2 horas \\
\hline Semana 4 & Evaluación & Evaluación final & 30 minutos \\
\hline
\end{tabular}

A continuación, se detalla el desarrollo de cada una de las tareas previstas:

1. Evaluación: se evaluará con un cuestionario de creación propia el conocimiento que los alumnos poseían en relación a los contenidos a tratar durante el CMD.

2. Estudio del temario: los estudiantes analizarán y reflexionarán sobre los contenidos a modo de lectura y estudio de los apuntes aportados por el profesor a través del aula virtual. Los alumnos contestarán a una serie de preguntas claves (Cuaderno de apuntes, Anexo I) que les ayudarán a conocer lo más relevante de cada contenido.

3. Resolución de dudas: en base a una lectura previa del cuaderno de apuntes, el profesor dirigirá un proceso de dudas y debate. 
4. Resolución de problema I y II: en esta actividad se resolverán dos problemas con el objetivo de ayudar a los estudiantes a ordenar conceptos e ideas relacionados con los medios y métodos de entrenamiento. 4.1. Problema I: Selecciona un contexto determinado en un entrenamiento de fuerza. ¿Qué medio de entrenamiento de fuerza crees que es más propio para su trabajo? ¿Crees que es posible trabajar otros objetivos con él? ¿Cuáles?

4.1.1. Este problema será resuelto en parejas. En esta fase del estudio, los estudiantes aún no habrán asimilado bien las diferencias entre medio y método de entrenamiento. Por ello, se espera que la mayoría hablen aquí de métodos de entrenamiento y no de medios. Sin embargo, esto no es un problema del todo, ya que a la hora de solucionar el problema será posible ir introduciendo diferentes métodos de entrenamiento. En relación a los objetivos, si se espera que los estudiantes sean capaces de conectar métodos con objetivos de entrenamiento de forma coherente, gracias a los contenidos vistos en temas anteriores.

4.2. Problema II: En base al contexto anterior, ¿qué método de entrenamiento crees que es más propicio para conseguir el objetivo expuesto anteriormente?

4.2.1. Este problema será resuelto en grupos de 4 personas (2 parejas juntas de la resolución anterior). En este punto, se espera que los alumnos recapaciten ahora sí sobre las diferentes entre método y medio. Con esto, se espera que se den cuenta de que antes, cuando se les preguntó por medios, hablaron de métodos. Por tanto, esta pregunta ya la habrían contestado en el problema anterior, debiendo ahora contestar sobre lo 
que se preguntaba en el problema anterior realmente, es decir, sobre los medios de entrenamiento.

5. Vivencias prácticas: práctica basada principalmente en contenidos procedimentales, donde el principal objetivo es afianzar los contenidos conceptuales observados en los supuestos prácticos y dudas expuestos previamente en el aula. Para su ejecución, algunos estudiantes del grupo organizarán una vivencia práctica basada en métodos y medios de entrenamiento, en relación a objetivos de entrenamiento determinados previamente. Concretamente, en la vivencia práctica I se realizarán tareas en relación con la mitad de los objetivos habituales del entrenamiento de fuerza (fuerza dinámica máxima y fuerza explosiva), utilizando medios de entrenamiento habituales para ello (medios concéntricos, pliométricos y sprint). En la vivencia práctica II se practicarán los siguientes objetivos de entrenamiento de fuerza (potencia máxima y resistencia a la fuerza), utilizando medios excéntricos, rotacionales y de resistencia variable. Se organizarán los ejercicios a modo de circuito de entrenamiento, donde los estudiantes plantearan diversas variantes y el profesor tendrá un rol de observación y feedback cuando así lo estime oportuno.

\section{Evaluación del CMD}

Para valorar el aprendizaje y evolución de los estudiantes durante este CMD, se utilizará el siguiente cuestionario. Son una serie de 4 preguntas abiertas, que pueden ser contestadas sin conocimientos técnicos previos. Dicho cuestionario se realizará de forma anónima en tiempo de clase:

1. Lista las diferentes formas (medios de entrenamiento) que conoces para poder entrenar fuerza 
con cualquier tipo de objetivo y metodología que se te ocurra.

2. El entrenamiento hasta el fallo muscular mejora la fuerza desde el punto de vista estructural y neural. Si tuvieras que elegir un medio de entrenamiento para este objetivo de la lista anterior, cuál sería y por qué.

3. También, se ha demostrado que no llegar al fallo muscular (método de esfuerzo submáximo) puede conseguir las mismas ganancias de fuerza neural que entrenar hasta el fallo muscular, además de otras ventajas. ¿Qué significa no llegar al fallo muscular y cómo indicarías un entrenamiento genérico con este objetivo? De la primera lista, ¿qué medio elegirías para ello?

4. María es una deportista de natación, en especialidad de $200 \mathrm{~m}$ libres. Aunque cuando va terminando su prueba va recuperando posiciones, en el inicio de la prueba suele quedarse atrás. ¿Por qué? Para mejorar esto, su entrenador le propone un ejercicio de fuerza. ¿Cómo crees que puede mejorar más, llegando al fallo muscular, haciendo muchas repeticiones llegando al fallo muscular o haciendo muchas repeticiones sin llegar al fallo muscular? ¿con qué medio de entrenamiento lo llevaría a cabo mejor y por qué?

\section{Aplicación del CMD}

\section{Relato de la aplicación}

Al comienzo del ciclo, en la evaluación inicial, se observó una gran sorpresa en los alumnos, aunque los sentimientos y las sensaciones fueron positivas. Sin embargo, algunos estudiantes mostraron cierta frustración por no resolver inmediatamente las preguntas y tener 
que esperar un tiempo hasta conocer las respuestas que yo recomiende. A medida que avanzó el ciclo, en la Resolución de dudas I, algunos alumnos comienzan a darse cuenta de la confusión que traían en terminologías y experiencias al leer los apuntes. Comienzan a conectar contenidos de temas anteriores, más teóricos, al ver un tema eminentemente de contenido más práctico y/o aplicable, aunque la mayoría siguen pensando en el medio antes que en el método o incluso el objetivo. Se observa que les cuesta trabajo asimilar algunos medios de entrenamiento por conceptos fisicos anteriores que debían saber, pero no traían aprendidos. En la Resolución del problema I, de repente, salta una chispa y comienzan a ver la incongruencia de pensar primer en el medio y luego en el objetivo. De hecho, casi todos hablan del método a la vez que del medio, aunque aún no ven que el medio es lo último y es independiente.

Tras solo unas horas, en la Vivencia práctica I, ya consiguen entender qué es un medio y qué es un método. Sin embargo, no consiguen aún diferenciar los métodos, aunque lo que más me preocupa es que no han asimilado las clasificaciones y tipos de objetivos que se vieron semanas anteriores. A pesar de que la metodología just in time que se viene aplicando desde hace unas semanas les está obligando a seguir un trabajo diario y, a expensas de que la sensación general del grupo es que es mucho trabajo y que solo dedican tiempo a esta asignatura, creo que aún no se han parado a leer en reflexión ni a estudiar la información, tan solo a realizar lecturas diagonales para cumplir en plazo las entregas, lo que no se si será productivo del todo.

En la Resolución de dudas II, los estudiantes siguen comentando, con mayor hincapié, que este tema es mucho más fácil que el anterior. Yo no lo veo así, sino que cada vez le van sonando mucho más los conceptos y términos específicos, a la vez que van asimilando el contenido. Durante 
la resolución del cuaderno de apuntes II, observo que ya se ha establecido normativo quiénes son aquellas personas que responden y quienes no, por lo que comienzo a cambiar mi pregunta de ¿quién puede resolverme esta pregunta? o ¿qué dificultades habéis tenido en esta pregunta? a preguntas concretas personales, enlazando con los deportes que se que practica cada uno, o bien con los temas que están desarrollando a nivel grupal en el trabajo final de la asignatura. Creo que los estudiantes han asimilado bien esta parte del tema y están preparados para, con algo más de estudio específico, poder comenzar a entrenar a deportistas en el entrenamiento de fuerza.

Al llegar a la Resolución del problema II les insté a recordar de la sesión anterior qué deporte y medio de entrenamiento habían elegido, para pensar ahora en el método de entrenamiento. En esta ocasión, en lugar de en parejas, al realizarlo en grupos de 4 personas, las interacciones entre los alumnos fueron más fructíferas al poder contrastar opiniones sobre dos casuísticas (una por pareja del problema anterior). Además, noto un mayor nivel de motivación en aquellos estudiantes que eligieron como contexto el propio tema del trabajo final de la asignatura. Para guiar la resolución del problema, coloqué en la pizarra los tipos de medios, objetivos y métodos posibles que estábamos manejando en clase. Así, iba preguntando a cada grupo que estableciesen las relaciones que ellos estimasen oportunas para sus situaciones específicas. De repente, se obró la magia: durante la resolución del problema, algunos estudiantes de repente comenzaron a hablar por el objetivo, luego el método y finalmente el medio, sin haber comentado yo nada al respecto de ese orden. Sin embargo, creo que no todos en este caso eran conscientes aún de ellos. El final de la resolución fue apoteósico. De forma genérica, comencé a preguntar. Al inicio hubo un interesante debate, ya que no terminaban de ver lo que vendría al final. Cuando terminamos todas las relaciones, se dieron cuenta que casi cualquier medio, vale para trabajar 
cualquier objetivo con cualquier método. Por ello, lancé la pregunta: "Si cualquier medio a través de cualquier método vale para trabajar cualquier objetivo, ¿por qué todos pensáis primero en cómo vais a hacer el entrenamiento y con qué (método y medio), antes de plantearos qué necesita el deportista (objetivo)? La cara de los estudiantes fue un poema, se habían dado cuenta de que había estado jugando con ellos durante dos semanas y guiándolos de un pensamiento no lógico hacia la lógica, casi sin haberse dado cuenta.

Por último, la Vivencia práctica II fue la actividad más fluida de todas. Conocido "el problema y truco" de este ciclo de mejora, la asimilación de los métodos y medios relacionados ya aquí fue mucho más fácil. Además, fue muy llevadera ya que uno de los medios es muy novedoso y casi nadie había practicado con el (medio rotacional).

\section{Resultados del aprendizaje}

La Tabla 2 muestra los resultados del aprendizaje al comparar los resultados de los cuestionarios realizados. Se observa una notable mejora del aprendizaje en el grueso de los estudiantes.

Tabla 2. Resultados del aprendizaje

\begin{tabular}{|c|c|c|c|c|c|c|c|c|c|c|c|c|c|c|c|c|c|c|c|c|}
\hline Pregunta & \multicolumn{9}{|c|}{1} & \multicolumn{1}{|c|}{2} & \multicolumn{7}{|c|}{3} & \multicolumn{5}{|c|}{4} \\
\hline Guntuación & 0 & A & B & C & D & 0 & A & B & C & D & 0 & A & B & C & D & 0 & A & B & C & D \\
\hline Pre (\%) & 5 & 23 & 17 & 5 & 0 & 9 & 23 & 17 & 2 & 0 & 2 & 23 & 23 & 0 & 3 & 4 & 25 & 13 & 7 & 0 \\
\hline Post (\%) & 0 & 0 & 3 & 0 & 47 & 0 & 1 & 9 & 22 & 17 & 0 & 4 & 9 & 20 & 18 & 1 & 12 & 12 & 14 & 13 \\
\hline Cambio (\%) & - & - & - & - & + & - & - & - & + & + & - & - & - & + & + & - & - & - & + & + \\
\hline & 5 & 23 & 15 & 5 & 47 & 9 & 21 & 7 & 21 & 17 & 2 & 19 & 14 & 20 & 15 & 3 & 13 & 1 & 7 & 13 \\
\hline
\end{tabular}

La Tabla 3 se muestra las evoluciones individuales de los alumnos. 
Tabla 3. Evoluciones individuales

\begin{tabular}{|c|c|c|c|c|c|c|c|c|}
\hline Alumno & PRE1 & PRE2 & PRE3 & PRE4 & POS1 & POS2 & POS3 & POS4 \\
\hline 1 & B & A & A & A & D & $C$ & $\mathrm{D}$ & B \\
\hline 2 & B & 0 & 0 & B & $\mathrm{D}$ & B & B & A \\
\hline 3 & 0 & 0 & B & A & $\mathrm{D}$ & B & $C$ & $C$ \\
\hline 4 & B & A & B & A & $\mathrm{D}$ & - & $\mathrm{D}$ & $\mathrm{D}$ \\
\hline 5 & $A$ & - & $A$ & C & D & $C$ & B & A \\
\hline 6 & - & $B$ & $B$ & $A$ & - & $C$ & $D$ & D \\
\hline 7 & 0 & 0 & $A$ & $A$ & $D$ & $C$ & D & B \\
\hline 8 & $B$ & $A$ & $B$ & $A$ & D & D & $C$ & D \\
\hline 9 & $A$ & $B$ & $A$ & $A$ & $D$ & B & - & $C$ \\
\hline 10 & A & B & B & B & $\mathrm{D}$ & $\mathrm{D}$ & $C$ & $C$ \\
\hline 11 & $A$ & A & B & 0 & B & A & $C$ & 0 \\
\hline 12 & $B$ & $A$ & $D$ & $A$ & $D$ & $C$ & D & D \\
\hline 13 & $A$ & $B$ & $A$ & $A$ & $D$ & $C$ & $C$ & B \\
\hline 14 & $B$ & 0 & $B$ & 0 & $D$ & D & $C$ & $A$ \\
\hline 15 & $A$ & $A$ & $B$ & $B$ & $D$ & B & B & A \\
\hline 16 & $A$ & A & $A$ & A & $\mathrm{D}$ & $\mathrm{D}$ & $\mathrm{D}$ & B \\
\hline 17 & C & $B$ & $A$ & $A$ & $D$ & $\mathrm{D}$ & D & $\mathrm{D}$ \\
\hline 18 & C & $B$ & $D$ & $B$ & D & D & C & B \\
\hline 19 & B & B & B & $C$ & $\mathrm{D}$ & $C$ & $C$ & C \\
\hline 20 & A & B & B & A & $\mathrm{D}$ & $C$ & $C$ & A \\
\hline 21 & $B$ & $A$ & $A$ & $A$ & D & B & B & D \\
\hline 22 & A & B & A & $C$ & B & $C$ & $C$ & A \\
\hline 23 & - & C & - & A & $\mathrm{D}$ & C & $C$ & $C$ \\
\hline 24 & $A$ & $A$ & $B$ & $C$ & $D$ & $D$ & D & C \\
\hline
\end{tabular}

*Leyenda de colores: Verde= evolución positiva. Gris= no evoluciona. Naranja= evolución negativa.

(1) 
La Figura 3 muestra un ejemplo de escalera de aprendizaje de la pregunta 4, la cual ha obtenido la menor evolución en la adquisición de los contenidos. Aunque ha supuesto la menor evolución, un cuarto de los estudiantes consiguieron llegar al último escalón, quedando más de la mitad en las zonas altas de la escalera.

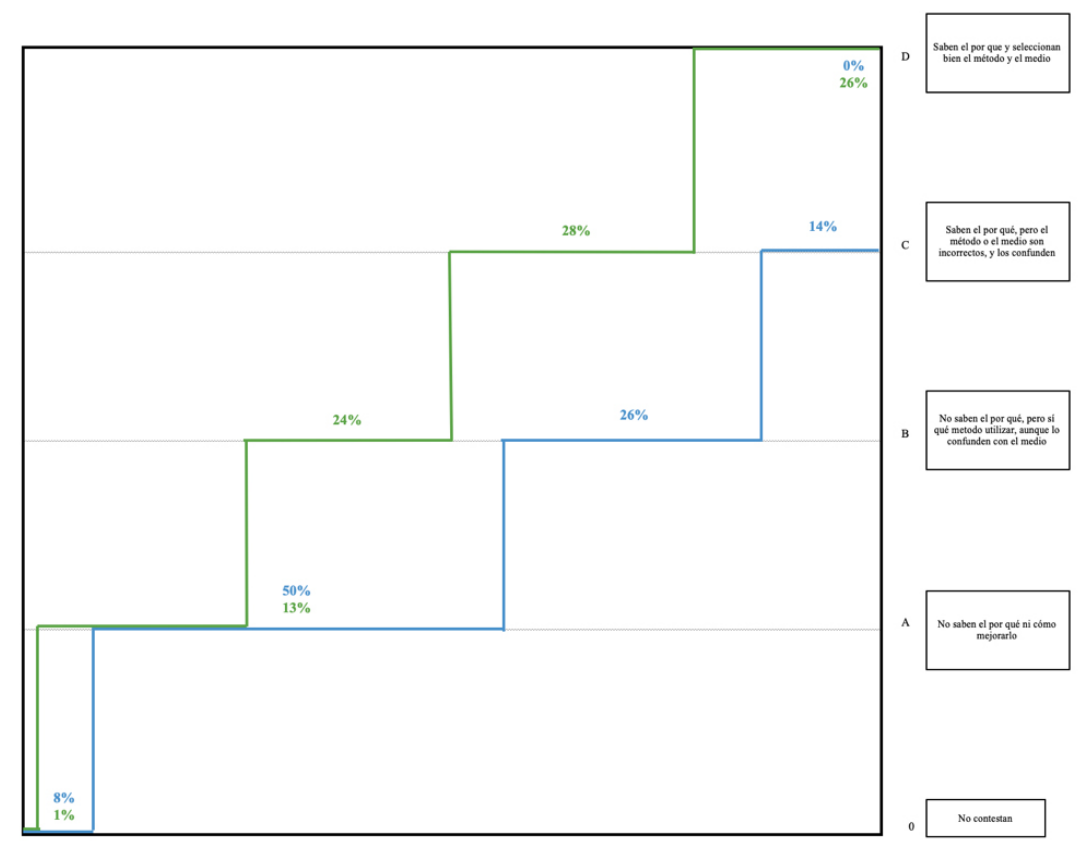

Figura 3. Ejemplo de escalera de aprendizaje de una de las preguntas del cuestionario de evaluación.

\section{Evaluación del CMD}

La incorporación de un CMD a mi enseñanza me ha ayudado a organizar mi docencia en diferentes pasos y utilizando diversos instrumentos para dar un dinamismo diferente a estas. Sin duda, lo más relevante que destaco va en línea de las enseñanzas del profesor Finkel (Finkel, 2008), en referencia a diseñar experiencias que enseñen a través de la secuenciación de preguntas con el profesor como tutor y observador del aprendizaje en un entorno creado. 
Antes de iniciar mi CMD, me costaba saber cómo llegar a evaluar realmente la adquisición de los diferentes contenidos. Incluso, quería buscar formas entretenidas de aprendizaje. La construcción de problemas y su resolución en clase era una de las estrategias que tenía en mente. Sin embargo, nunca se me hubiera pasado por la cabeza comprender que es posible orientar las evaluaciones hacia la construcción de escaleras de aprendizaje donde poder encontrar los diferentes nudos y complicaciones donde los estudiantes presentan sus propias dificultades. Para ello, fue relevante el diseño de los cuestionarios, los cuales deben permitir una respuesta abierta y posible de ser resulta con cualquier tipo de conocimiento, como podría ser la interpretación de un hecho (Rivero \& Porlan, 2017).

De toda esta experiencia, por tanto, destaco la estrategia del análisis de los contenidos a modo de mapa conceptual, el cual seguiré utilizando en la misma medida que he aplicado, ya que he observado que de hacerlo mucho más detallado, no podría compartirlo bien con los alumnos, pues así se hizo. Aun así, me ayudó a plantear el diseño del CMD. La creación de entornos de aprendizaje es sin duda el primer objetivo que seguiré trabajando en cada uno de los contextos en los que interactúe: no sé si llegaré al nivel de crear un CMD por cada bloque de contenidos, pero sin duda utilizaré la resolución de problemas guiada a través de cuestionarios de una forma u otra para intentar crear escaleras de aprendizaje y observar los posibles nudos, tanto para los estudiantes donde se aplique, como para venideros.

Por último, en base a todo lo comentado, los principios didácticos en los que me he basado y seguro seguiré aplicando son:

1) Entender al alumnado y su forma de pensar desde un plano integral, intentando enlazar sus experiencias pasadas con nuevos aprendizajes. 
2) Organizar los contenidos en base a resolución de problemas ligados con experiencias pasadas 0 bien, crear entornos de aprendizaje que faciliten la adquisición de experiencias en tiempo real en relación con los bloques de contenidos que se estén impartiendo.

3) Analizar el proceso de enseñanza-aprendizaje no solo desde el conocimiento técnico-científico, sino desde la capacidad de razonar situaciones reales con los aprendizajes adquiridos.

4) Propiciar entornos de aprendizaje con trabajos en grupo, donde el diseño es primordial para asegurar que todo el grupo aporte algo, no ya en mayor o menor cantidad de trabajo, sino en la adquisición de roles concretos.

5) Entender los nudos de aprendizaje como un proceso natural en la asimilación de contenidos, basando el tiempo y actividades que se dediquen en la asignatura principalmente en ellos.

\section{Referencias bibliográficas}

Finkel, D. (2008). Experiencias que enseñan: crear esquemas para el aprendizaje. In D. Finkel (Ed.), Dar clase con la boca cerrada (1st ed., pp. 153-184). Universitat de Valencia. Servei de publications.

Prieto Martin, A., Diaz Martin, D., Monserrat Sanz, J., Alvarez-Mon Soto, M., Sanvicen Torne, P., \& Italo Rinaldi, M. (2018). Las tecnologías de la información y la comunicación como herramientas mediadoras de los procesos educativos. In M. Occelli, L. García Romano, N. Valeiras, \& M. Quintanilla Gatica (Eds.), Las tecnologías de la información y la comunicación como herramientas mediadoras de los procesos educativos (1st ed., pp. 117-128). Bellaterra Ltda.

Rivero, A., \& Porlan, R. (2017). La evaluación en la enseñanza universitaria. In R. Porlan (Ed.), Enseñanza Universitaria. Cómo mejorarla. (1st ed., pp. 73-88). Ediciones Morata. 


\section{Anexo I - Cuaderno de apuntes}

1. Utilidades de los medios isométricos en el entrenamiento de fuerza. Aplicaciones prácticas en el entrenamiento.

2. Tipos de medios isoinerciales y aplicaciones prácticas para trabajar cada manifestación de la fuerza y/o objetivo de fuerza específico.

3. Qué aporta un medio rotacional de resistencia variable en comparación con un peso libre.

4. De qué forma los medios de resistencia neumática y de resistencia variable pueden suponer un plus en el entrenamiento de fuerza. Situaciones específicas en las que podrían ser contraproducentes.

5. Diferencias principales entre los medios pasivos y los medios activos en el entrenamiento de fuerza. Utilidades y efectos comprobados de la ciencia.

6. Tipos de métodos genéricos de entrenamiento de fuerza según el tipo de esfuerzo realizado.

7. Caracteristicas del continuum de fuerza, en relación al volumen, intensidad, tiempo de descanso y tipos de objetivos o targets habituales del entrenamiento de fuerza.

8. Ventajas y desventajas en la utilización del método de esfuerzos submáximos y el método de esfuerzos repetidos. Relación con el carácter del esfuerzo y la fatiga que produce cada relación.

9. Diferencias principales entre los métodos pliométricos y métodos balísticos

10. Relación entre la velocidad sprint y fuerza. ¿Existe el entrenamiento de la velocidad? ¿Cómo se mejora la velocidad de desplazamiento? 